\title{
Corrigendum
}

\section{Corrigendum to "Control of a Humanoid NAO Robot by an Adaptive Bioinspired Cerebellar Module in 3D Motion Tasks"}

\author{
Alberto Antonietti $\mathbb{D}^{1},{ }^{1}$ Dario Martina $\left(\mathbb{D},{ }^{1}\right.$ Claudia Casellato ${ }^{(D)},{ }^{2}$ Egidio D'Angelo $\left(\mathbb{D},{ }^{2,3}\right.$ \\ and Alessandra Pedrocchi ${ }^{1}{ }^{1}$ \\ ${ }^{1}$ Department of Electronics, Information and Bioengineering, Politecnico di Milano, Milano, Italy \\ ${ }^{2}$ Department of Brain and Behavioral Sciences, University of Pavia, Pavia, Italy \\ ${ }^{3}$ IRCCS Mondino Foundation, Pavia, Italy \\ Correspondence should be addressed to Alberto Antonietti; alberto.antonietti@polimi.it \\ Received 15 October 2020; Accepted 15 October 2020; Published 23 October 2020 \\ Copyright (c) 2020 Alberto Antonietti et al. This is an open access article distributed under the Creative Commons Attribution \\ License, which permits unrestricted use, distribution, and reproduction in any medium, provided the original work is \\ properly cited.
}

In the article titled "Control of a Humanoid NAO Robot by an Adaptive Bioinspired Cerebellar Module in 3D Motion Tasks" [1], there was an error in Dr. Egidio D'Angelo's affiliation. The corrected authors list and affiliations are shown above.

\section{References}

[1] A. Antonietti, D. Martina, C. Casellato, E. D’Angelo, and A. Pedrocchi, "Control of a humanoid NAO Robot by an adaptive bioinspired cerebellar Module in 3D motion Tasks," Computational Intelligence and Neuroscience, vol. 2019, Article ID 4862157, 15 pages, 2019. 